\title{
Graphene and black holes: novel materials to reach the unreachable
}

\author{
Alfredo lorio* \\ Faculty of Mathematics and Physics, Charles University, Prague, Czech Republic \\ ${ }^{*}$ Correspondence: alfredo.iorio@mff.cuni.cz \\ Edited by: \\ Simone Taioli, Fondazione Bruno Kessler, Italy \\ Reviewed by: \\ Alessio Paris, Fondazione Bruno Kessler, Italy
}

Keywords: graphene, quantum field theory, black holes, hawking radiation, quantum gravity

Physics is an experimental science. Nonetheless, in the last decades, it proved very difficult (if not impossible) to reconcile theoretical investigations of the fundamental laws of Nature with the necessary experimental tests. The divarication between theory and experiments is the central problem of contemporary fundamental physics that, to date, is still unable to furnish a consistent quantum theory of gravity, or to obtain experimental evidences of milestones theories like Supersymmetry [see, e.g., the whole Issue (de Haro et al., 2013) on the status of String Theory].

A widespread view of this problem is that experimental observations of these types of phenomena can only be achieved at energies out of the reach of our laboratories (e.g., the Planck energy $10^{19} \mathrm{GeV}$ vs. reachable energies in our laboratories of $10^{3} \mathrm{GeV}$ ). In our view, due to unprecedented behaviors of certain novel materials, nowadays indirect tests should be considered as a viable alternative to direct observations.

This field of research is not a novelty. It usually goes under the generic name of "analog gravity" (Novello et al., 2002; Volovik, 2003; Barceló et al., 2011), although, perhaps, to call it "bottom-up" approach does it more justice: analog experimental settings on the bottom, fundamental theories of Nature on the top. Nonetheless, for various reasons, it has been seen as little more than a curiosity. It is seen as an amusing and mysterious series of coincidences that (to our knowledge) never are taken as tests of the aspects of the fundamental theories they reproduce. Neither is taken as experimental tests of the fundamental theories, the mysterious and amusing coincidences of the "top-bottom" approach of the AdS/CFT correspondence [where theoretical constructions of the fundamental world are used to describe experimental results at our energy scale (Maldacena, 2012)].

As well known, with graphene (Novoselov et al., 2004), we have a quantum relativistic-like Dirac massless field available on a nearly perfectly two-dimensional sheet of carbon atoms [see Castro Neto et al. (2009) for a review]. Recent work shows the emergence of gravity-like phenomena on graphene (Iorio, 2011, 2012, 2013; Iorio and Lambiase, 2012, 2014). More precisely, the Hawking effect can take place on graphene membranes shaped as Beltrami pseudospheres of suitably large size, hence even $(2+1)$-dimensional black-hole scenarios (Bañados et al., 1992) are in sight. The Hawking effect here manifests itself through a finite temperature electronic local density of states [for reviews, see Iorio $(2014,2015)]$.

The predictions of the Hawking effect on graphene are based on the possibility to obtain very specific shapes, e.g., the Beltrami pseudosphere that should recreate, for the pseudoparticles of graphene, conditions related to those of a spacetime with an horizon. What we are first trying (Gabbrielli et al., under preparation) is to obtain a clear picture of what happens to $\mathrm{N}$ classical particles, interacting via a simple potential, e.g., a Lennard-Jones potential, and constrained on the Beltrami. This will furnish important pieces of information on the actual structure of the membrane. In fact, is well known from similar work with the sphere [that goes under the name of "generalized Thomson problem," see, e.g., Bowick et al. (2000)] that defects will form more and more, and their spatial arrangements are highly non-trivial, and follow patterns related to the spontaneous breaking of the appropriate symmetry group (Iorio and Sen, 2006). Once the coordinates of the $N$ points are found in this way, we need to simulate the behaviors of Carbon atoms arranged in that fashion, hence, we essentially change the potential to the appropriate one, and perform a Density Functional type of computation. The number of atoms that we can describe this way is of the order of $10^{3}$, highly demanding computer-time wise, but still too small for the reaching of the horizon. Nonetheless, the results obtained will be important to refine various details of the theory. We need to go further, toward a big radius of curvature $r$, when the Hawking effect should be visible.

Any serious attempt to understand Quantum Gravity has to start from the Hawking effect. That is why black holes are at the crossroad of many of the speculations about the physics at the Planck scale. From (Iorio, 2011, 2012, 2013; Iorio and Lambiase, 2012, 2014) a goal that seems in sight is the realization of reliable set-ups, where graphene well reproduces the blackhole thermodynamics scenarios, with the analog gravity of the appropriate kind to emerge from the description of graphene's membrane. The lattice structure, the possibility to move through energy regimes where discrete and continuum descriptions coexist, and the unique features of matter fields whose relativistic structure is induced by the spacetime itself, are all issues related to Quantum Gravity (Loll, 1998; 't Hooft, 2009) that can be explored with graphene.

Many other tantalizing fundamental questions can be addressed with graphene. 
To mention only two: there are results of Alvarez et al. $(2012,2014)$ that point toward the use of graphene to have alternative realizations of Supersymmetry, and there are models of the Early Universe, based on $(2+1)$-dimensional gravity (van der Bij, 2007), where graphene might also play a role.

We are lucky that these "wonders" are predicted to be happening on a material that is, in its own right, enormously interesting for applications. Hence, there is expertise worldwide on how to manage a variety of cases. Nonetheless, the standard agenda of a material scientist is of a different kind than testing fundamental laws of Nature. Therefore, let us conclude by invoking the necessity of a dedicated laboratory, where condensed matter and other low energy systems are experimentally studied with the primary goal of reproducing phenomena of the fundamental kind.

For the reasons outlined above, graphene is a very promising material for this purpose.

\section{ACKNOWLEDGMENTS}

The author warmly thanks the LISC laboratories of Fondazione Bruno Kessler and $\mathrm{ECT}^{\star}$, Trento, Italy, for the kind hospitality. Funding: the author acknowledges the Czech Science Foundation (GAČR), Contract No. 14-07983S, for support.

\section{REFERENCES}

de Haro, S., Dieks, D., 't Hooft, G., and Verlinde, E. (eds). (2013). Forty years of string theory: reflecting on the foundations. Found. Phys. 43(1). Available at: http://link.springer.com/ journal/10701/43/1/page/1
Alvarez, P. D., Pais, P., and Zanelli, J. (2014). Unconventional supersymmetry and its breaking. Phys. Lett. B 735, 314-321. doi:10.1016/j.physletb.2014.06.031

Alvarez, P. D., Valenzuela, M., and Zanelli, J. (2012). Supersymmetry of a different kind. J. High Energy Phys. 2012:058. doi:10.1007/JHEP04(2012)058

Bañados, M., Teitelboim, C., and Zanelli, J. (1992). The black hole in three-dimensional spacetime. Phys. Rev. Lett. 69, 1849-1851. doi:10.1103/PhysRevLett. 69.1849

Barceló, C., Liberati, S., and Visser, M. (2011). Analogue gravity. Liv. Rev. Rel. 14, 3. doi:10.12942/lrr2011-3

Bowick, M. J., Nelson, D. R., and Travesset, A. (2000). Interacting topological defects on frozen topographies. Phys. Rev. B 62, 8738-8751. doi:10.1103/ PhysRevB.62.8738

Castro Neto, A. H., Guinea, F., Peres, N. M. R., Novoselov, K. S., and Geim, A. K. (2009). The electronic properties of graphene. Rev. Mod. Phys. 81, 109-162. doi:10.1103/RevModPhys.81.109

Iorio, A. (2011). Weyl-gauge symmetry of graphene. Ann. Phys. 326, 1334-1353. doi:10.1016/j.aop.2011. 01.001

Iorio, A. (2012). Using Weyl symmetry to make graphene a real lab for fundamental physics. Eur. Phys. J. Plus 127:156. doi:10.1140/epjp/i201212156-1

Iorio, A. (2013). Graphene: QFT in curved spacetimes close to experiments. J. Phys. Conf. Series 442, 012056. doi:10.1088/1742-6596/442/1/012056

Iorio, A. (2014). Weyl Symmetry in Graphene, Lecture Notes at the Workshop "Modeling Graphene-like Systems". University College Dublin. Available at: http://mathsci.ucd.ie/ miguel/GRAPHENE_ WORKSHOP_2014/Alfredo_Iorio/

Iorio, A. (2015). Curved spacetimes and curved graphene: a review of the Weyl-symmetry approach. Int. J. Mod. Phys. D (in press).

Iorio, A., and Lambiase, G. (2012). The HawkingUnruh phenomenon on graphene. Phys. Lett. B 716, 334-337. doi:10.1016/j.physletb.2012.08.023

Iorio, A., and Lambiase, G. (2014). Quantum field theory in curved graphene spacetimes, Lobachevsky geometry, Weyl symmetry, Hawking effect, and all that. Phys. Rev. D 90, 025006. doi:10.1103/ PhysRevD.90.025006
Iorio, A., and Sen, S. (2006). Topological constraints on the charge distributions for the Thomson problem. Phys. Rev. B 74, 052102. doi:10.1103/PhysRevB.74. 052102

Loll, R. (1998). Discrete approaches to quantum gravity in four dimensions. Liv. Rev. Rel. 1:13. doi:10. 12942/lrr-1998-13

Maldacena, J. (2012). "The gauge/gravity duality," in Black Holes in Higher Dimensions, ed. G. T. Horowitz (Cambridge: Cambridge University Press).

Novello, M., Visser, M., and Volovik, G. E. (2002). Artificial Black Holes. Singapore: World Scientific.

Novoselov, K. S., Geim, A. K., Morozov, S. V., Jiang, D., Zhang, Y., Dubonos, S. V., et al. (2004). Electric field effect in atomically thin carbon films. Science 306, 666-669. doi:10.1126/science.1102896

van der Bij, J. J. (2007). Cosmotopological relation for a unified field theory. Phys. Rev. D 76, 121702. doi:10.1103/PhysRevD.76.121702

Volovik, G. E. (2003). The Universe in a Helium Droplet. Oxford: Clarendon Press.

't Hooft, G. (2009). Cristalline gravity. Int. J. Mod. Phys. A 24,3243-3255. doi:10.1142/S0217751X09046849

Conflict of Interest Statement: The author declares that the research was conducted in the absence of any commercial or financial relationships that could be construed as a potential conflict of interest.

Received: 04 December 2014; accepted: 18 December 2014; published online: 12 January 2015.

Citation: Iorio A (2015) Graphene and black holes: novel materials to reach the unreachable. Front. Mater. 1:36. doi: 10.3389/fmats.2014.00036

This article was submitted to Mechanics of Materials, a section of the journal Frontiers in Materials.

Copyright (c) 2015 Iorio. This is an open-access article distributed under the terms of the Creative Commons Attribution License (CC BY). The use, distribution or reproduction in other forums is permitted, provided the original author(s) or licensor are credited and that the original publication in this journal is cited, in accordance with accepted academic practice. No use, distribution or reproduction is permitted which does not comply with these terms. 